\title{
MARKOV CHAINS AND THE DETERMINATION OF FAIR PREMIUMS
}

\author{
STEFAN VAJDa \\ Birmingham
}

The relationships between actuarial and pure mathematics are curious. Actuaries have contributed to the development of mathematical theory: it is sufficient to mention, as examples, Fredholm of an earlier, and Cramér of a more recent generation. Scandinavian mathematicians, in particular, have been concerned with a very special type of stochastic process, reflected in the collective theory of risk, and the work of Philipson, Ammeter and others in this field is well known to readers of this Bulletin. However, the main stream of the theory of stochastic processes has little contact with actuarial applications.

On the other hand, many actuaries have studied and assimilated pure mathematics and have thrown light on actuarial matters by describing their own preoccupations in the terminology of modern, often abstract, mathematics. E. Franckx is one of their number.

The Instituto di Matematica Finanziaria of the University of Trieste (Faculty of Economics and Commerce) has published a booklet entitled

Essai d'une théorie opérationnelle des risques Markoviens which contains three lectures delivered by Professor Franckx in Trieste and a contribution which he presented to the 17 th Congress of Actuaries, held in London in I964.

The central concept in these lectures is that of a Markov chain, a special stochastic process. It is assumed that, at any given time, an item can be in any one of $n$ states, and that the probabilities of passing, within the next unit of time, from state $i$ (provided it is now there) to state $j$ are known. They are denoted by $p_{i j}$. The matrix $\left(p_{i j}\right)$ of these probabilities is referred to as a Markov matrix, or a transition matrix.

It is easily seen that the transitions of individuals aged $x$ into the state of being of age $x+\mathrm{I}$, or into the state of having died can 
be described in terms of Markov chains. (The last named state is 'absorbing', i.e. the probability of remaining in that state is unity, and that of passing into another state is zero.)

This is discussed in the first lecture, where liabilities and premiums are introduced as payments connected with the transitions: typically, a person aged $x$ gives up his premium reserve and pays a premium in order to receive either the premium reserve of the next higher age, with probability $p_{x}$, or the sum assured with probability $q_{x}$.

The second lecture extends these ideas to more general risks, taking into account, for instance, transitions from the active to the disabled state, with varying probabilities dependent on age.

Lecture No. 3 deals with non-life assurance. In many ways this is a simpler case than that of life assurance. But it is here where we find the investigation which might be considered the core of this course of lectures.

To introduce the problem, we might think of motor insurance. An underwriter who accepts a particular risk has only incomplete information on which to base the computation of a fair premium. $\mathrm{He}$ will, in many cases, charge the same premium for risks which, after one year's experience, may turn out to belong into quite different categories.

Let us now assume that we know the values $p_{i j}$ which describe the probabilities that a risk which, in a given year, has produced a claim level $i$, will during the next year produce a claim level $j$. The underwriter will place his risks into different categories, dependent on the first year's claim level. Which premium should he now charge? If it is, for instance, not unlikely that a risk with high claims in the first year may show low claims in later years, then one might suspect that it is, after all, fair to charge the same premium to all categories (i.e. whatever the experience, perhaps misleading, of the first year).

To fix our ideas, let us assume that the underwriter places his risks into two categories, according to the experience in the first year, which might have shown a claim $C_{1}$ for those in the first, and of $C_{2}$ for those in the second category. Let the known transition probabilities between claim experiences be as follows: 


$\begin{array}{crrr} & & \text { claim } C_{1} & C_{2} \text { next year } \\ \text { claim this } & C_{1} & \mathrm{I} / 3 & 2 / 3 \\ \text { year } & C_{2} & 3 / 4 & \mathrm{I} / 4\end{array}$

As a numerical illustration we shall assume that $C_{1}=o$ and $C_{2}$ $=\mathrm{I}$. Then a risk belonging into category $\mathrm{I}$ should, for the next year, be charged a premium of $C_{1 / 3}+2 C_{2} / 3=2 / 3$, say, and a risk in the second category should be charged a premium of $3 C_{1} / 4$ $+C_{2} / 4=\mathrm{I} / 4$, say.

Let us now compute the premium for the third year. It is $(\mathrm{I} / 3 \times \mathrm{I} / 3+2 / 3 \times 3 / 4) C_{1}+(\mathrm{I} / 3 \times 2 / 3+2 / 3 \times \mathrm{I} / 4) C_{2}=7 / \mathrm{I} 8$ say for the first category, and

$(3 / 4 \times \mathrm{r} / 3+\mathrm{I} / 4 \times 3 / 4) C_{1}+(3 / 4 \times 2 / 3+\mathrm{I} / 4 \times \mathrm{I} / 4) C_{2}=9 / \mathrm{I} 6$, say for the second. The coefficients are the probabilities that claim $C_{1}$ or $C_{2}$ will become due for the respective categories. In Matrix notation we can write the premium for the second year as the rows of the matrix product

$$
\left(\begin{array}{ll}
1 / 3 & 2 / 3 \\
3 / 4 & \mathrm{I} / 4
\end{array}\right) \quad\left(\begin{array}{l}
C_{1} \\
C_{2}
\end{array}\right)=M . C, \text { say }
$$

and those for the year after that as the rows of

$$
\left(\begin{array}{ll}
\mathrm{I} / 3 & 2 / 3 \\
3 / 4 & \mathrm{I} / 4
\end{array}\right) \quad\left(\begin{array}{ll}
\mathrm{I} / 3 & 2 / 3 \\
3 / 4 & \mathrm{I} / 4
\end{array}\right) \quad\left(\begin{array}{l}
C_{1} \\
C_{2}
\end{array}\right) \quad=M^{2} . C
$$

It emerges that the fair premium for the $t$-th year after the first year will be given by the rows of the matrix product $M^{t} . C$.

We notice that the premiums for the two categories, viz. $2 / 3$ and $I / 4$ in the second, and $7 / 18$ and $9 / 16$ in the third year and so on get closer as the years proceed. Franckx proves-rather neatlythat this concentration is always the case if the matrix $M$ has row totals I, which is always the case for Markov matrices. He then asks under what conditions the powers of such a matrix converge to a matrix whose rows are all equal. This is an important question, because if this is so, then all categories will, in the long run, be charged identical premiums, and Franckx advances this as a justification for all risks to be charged identical premiums ab initio, when their risk structure is not yet known. The risks attaching to the categories with such a Markov matrix are called 'normal'. 
It is known (from a theorem due to Frobenius) that the powers of a Markov matrix converge to a matrix with identical rows if there exists a power $M^{k}$ (with finite $k$ ) such that all its elements are positive (and none is zero).

It is natural to ask here how one recognizes whether a given matrix has such a power. The author points out that this is certainly the case if $P_{1 j}>0$ for all $j$ and also $p_{i \mathbf{1}}>0$ for all $i$. He calls a risk 'good' if, moreover, $p_{11}$ is large.

The risk level denoted by $I$ is that where no claim is to be paid. It follows easily that all 'good' risks are 'normal'.

It will be noticed that the computation of premiums depends, in this study, on expected values. No matters appertaining to the theory of risk are touched upon.

The fourth lecture extends these considerations to the case of non-stationary matrices, i.e. those whose elements $p_{j k}$ change with time.

Unfortunately, the publication contains a number of misprints which make it difficult to read. However, the reviewer is confident that he has correctly presented Professor Franckx's interesting adaptation of the theory of positive non-decomposable matrices to a topical actuarial problem. 